\title{
The neuroblastoma and ganglion components of nodular ganglioneuroblastoma are genetically similar: evidence against separate clonal origins
}

\author{
Paola Angelini ${ }^{1}$, Sylvain Baruchel ${ }^{1,2}$, Paula Marrano ${ }^{3}$, Meredith S Irwin ${ }^{1,2}$ and \\ Paul S Thorner ${ }^{3,4}$ \\ ${ }^{1}$ Division of Haematology/Oncology, The Hospital for Sick Children, University of Toronto, Toronto, ON, \\ Canada; ${ }^{2}$ Department of Pediatrics, University of Toronto, University of Toronto, Toronto, ON, Canada; \\ ${ }^{3}$ Division of Pathology, The Hospital for Sick Children, Toronto, ON, Canada and ${ }^{4}$ Laboratory Medicine and \\ Pathobiology, University of Toronto, Toronto, ON, Canada
}

\begin{abstract}
Nodular ganglioneuroblastoma is characterized by a macroscopic nodule of neuroblastoma within a ganglioneuromatous component. These two components have been considered to originate from separate clones, with the neuroblastoma clone accounting for the clinical behavior of nodular ganglioneuroblastoma. In order to investigate the clonal origin of the cellular components (neuroblasts, ganglion cells, and Schwann cells) of nodular ganglioneuroblastoma, paraffin-embedded tumor samples from eight cases were analyzed by single nucleotide polymorphism array and in situ hybridization. DNA was extracted separately from neuroblastomatous and ganglioneuromatous areas. By in situ hybridization, MYCN gain (4-10 gene copies/ nucleus) was detected in 7/8 neuroblastoma samples. In ganglioneuromatous regions, gains were also detected in ganglion cells but not in Schwann cells. Single-nucleotide polymorphism array studies identified chromosome losses (11q and 14q) and gains (12, 13q, 17q and 18q) in the neuroblastoma component, whereas the ganglioneuromatous component showed fewer or no genetic alterations. There were no unique copy number changes distinguishing nodular ganglioneuroblastoma from other subtypes of neuroblastoma. By in situ hybridization, ganglion cells but not Schwann cells showed the same alterations detected in neuroblasts. Thus, neuroblasts and ganglion cells in nodular ganglioneuroblastoma are genetically related and may arise from the same clone. In contrast, the Schwann cells have a different origin and may be derived from a nonneoplastic neural crest precursor. Our results suggest that the clinical behavior of nodular ganglioneuroblastoma cannot be explained by the presence of separate clones with distinct genetic signatures. Modern Pathology (2015) 28, 166-176; doi:10.1038/modpathol.2014.90; published online 1 August 2014
\end{abstract}

Neuroblastoma, a malignancy of neural crest cells of the autonomic nervous system, is the most common extracranial solid tumor of childhood. ${ }^{1}$ The incidence is $\sim 1$ in 7000 children, with a median age at presentation of 17 months, ${ }^{2}$ and accounts for $15 \%$ of cancer-related deaths in childhood.1,3 A feature distinguishing neuroblastoma from most other pediatric malignancies is the striking heterogeneity of histology, biology, and clinical behavior. ${ }^{3,4}$ Features

Correspondence: Dr PS Thorner, MD, PhD, Division of Pathology, The Hospital for Sick Children, 555 University Avenue, Toronto, ON, Canada M5G 1X8.

E-mail: paul.thorner@sickkids.ca

Received 31 January 2014; revised 18 April 2014; accepted 19 April 2014; published online 1 August 2014 associated with a poor outcome include: age $>18$ months, ${ }^{2}$ unfavorable histopathology, ${ }^{5}$ amplification of the MYCN oncogene, ${ }^{6-8}$ diploid DNA content, and specific segmental chromosomal aberrations such as loss of heterozygosity at $1 \mathrm{p}$ and $11 \mathrm{q}$, gain of $17 \mathrm{q} \cdot{ }^{3,9}$

Currently, the pathology of neuroblastoma is graded according to the International Neuroblastoma Pathology Classification ${ }^{10-12}$ that separates histologic subtypes of neuroblastoma into favorable and unfavorable categories. One uncommon subtype is nodular ganglioneuroblastoma, which represents $5-10 \%$ of neuroblastic tumors. ${ }^{12-14}$ Nodular ganglioneuroblastoma is a composite tumor, with a predominance of differentiated Schwannian-type stroma associated with one or more macroscopically visible 
nodules of neuroblastoma showing neuropil formation but lacking this Schwannian stroma (referred to as 'stroma-poor'). Less common variants without visible nodules have more recently been included under the category of nodular ganglioneuroblastoma. ${ }^{15,16}$ In the original version of this classification system, this subtype was categorized with the unfavorable histology group, and those patients had a $46.8 \%$ event-free survival and $59.1 \%$ overall survival. ${ }^{12}$ More recently, it was shown that nodular ganglioneuroblastoma can be subcategorized as favorable or unfavorable according to the histopathology of the stroma-poor component, using the same criteria as for other stroma-poor neuroblastomas. ${ }^{15,16}$ By this system, unfavorable tumors outnumber favorable ones $\sim 2: 1$, with an event-free survival of $29-32 \%$ for unfavorable nodular ganglioneuroblastoma compared with $86 \%$ for favorable, and overall survival of $33-40 \%$ and $90-95 \%$, respectively. ${ }^{15,16}$ Subsequent studies have confirmed these clinical differences. ${ }^{13,17-19}$

Neuroblastoma is characterized by multiple chromosomal abnormalities (reviewed in refs 1,3), but whether these are shared by the nodular ganglioneuroblastoma subtype is not known. Genome-wide approaches such as array comparative genomic hybridization ${ }^{20-25}$ and single-nucleotide polymorphism arrays ${ }^{26,27}$ have been used to identify whole chromosome and segmental gains and losses in neuroblastoma; however, only two studies specifically mentioned the inclusion of ganglioneuroblastoma samples ${ }^{28,29}$ and results were inconsistent. The neuroblastoma component is thought to have a clonal origin distinct from the ganglioneuromatous part of the tumor. ${ }^{11,16}$ However, there is only one report in which the neuroblastic and ganglioneuromatous components were compared by array comparative genomic hybridization. ${ }^{30}$ In this study of six tumors, variable copy number alterations were detected in the neuroblastic component but not in the stroma-rich component, supporting the concept that these two components might be genetically distinct. To examine this issue further and specifically compare the genetic changes in the neuroblasts, ganglion, and Schwann cells, we used a combined approach of single-nucleotide polymorphism array and in situ hybridization. The goal of our study was to determine whether the different cellular components of nodular ganglioneuroblastoma have distinct chromosomal alterations and identify changes potentially specific to nodular ganglioneuroblastoma.

\section{Materials and methods}

\section{Study Group}

Between 1987 and 2008, there were eight patients diagnosed with nodular ganglioneuroblastoma at the Hospital for Sick Children, Toronto, Ontario, for which tumor tissue was available (Table 1). An additional 18 cases were identified but rejected for one of the following reasons: (1) insufficient tissue was available for the studies; (2) the diagnosis was based solely on a post-treatment histology and chemotherapy can alter the original histologic appearance to resemble nodular ganglioneuroblastoma; (3) review of the pathology failed to confirm the subtype of nodular ganglioneuroblastoma. The eight cases included six males and two females, ranging in age from 8 months to 7 years. The sites included adrenal gland, posterior mediastinum, and abdomen. Using International Neuroblastoma Risk Group criteria, six patients were categorized as low risk (one stage 1 and five stage 2) and two patients were high risk (both stage 4). Using the histologic grading system of the International Neuroblastoma Pathology Committee, ${ }^{15,16}$ four cases were categorized as favorable and four cases as unfavorable. Seven of eight patients had at least 4 years of followup. Two patients died of disease, one of which had stage 4 disease at diagnosis and the other was stage 2 at diagnosis but relapsed with distant metastatic disease. Paraffin-embedded tissue was available for

Table 1 Clinical features of nodular ganglioneuroblastoma study group

\begin{tabular}{|c|c|c|c|c|c|c|c|c|c|}
\hline Case & Age & Sex & Site & Stage & Risk & Histology & $\begin{array}{c}\text { Chemo before } \\
\text { biopsy }\end{array}$ & Met & Outcome \\
\hline 1 & 5 Years & M & Adrenal & 2 & Low $^{\mathrm{a}}$ & Diff, low MKI (unfavorable) + GNB intermixed & & & Alive \\
\hline 2 & 10 Months & $\mathrm{M}$ & Thoracic & 2 & Low & Poorly diff, inter MKI (unfavorable) + GNB intermixed & & & Alive \\
\hline 3 & 7 Years & $\mathrm{M}$ & Adrenal & 4 & High & Diff, low MKI (unfavorable) + GN maturing & $\mathrm{Y}$ & $\mathrm{Y}$ & Alive \\
\hline 4 & 2 Years & $\mathrm{M}$ & Thoracic & 2 & Low $^{\mathrm{a}}$ & Poorly diff, high MKI (unfavorable) + GN maturing & & Y & Dead \\
\hline 5 & 2 Years & $\mathrm{F}$ & Adrenal & 1 & Low & Diff, low MKI (favorable) + GNB intermixed & & & Alive \\
\hline 6 & 4 Years & $\mathrm{M}$ & Adrenal & 4 & High & Diff, low MKI (favorable) + GNB intermixed & $\mathrm{Y}$ & & Dead \\
\hline 7 & 8 Months & $\mathrm{F}$ & Thoracic & 2 & Low & Poorly diff, low MKI (favorable) + GN maturing & & & Alive \\
\hline 8 & 16 Months & $\mathrm{M}$ & Abdomen & 2 & Low & Diff, inter MKI (favorable) + GN maturing & & & Alive \\
\hline
\end{tabular}

Abbreviations: Diff, differentiated; Poorly diff, poorly differentiated; MKI, mitotic-karyorrhectic index; Inter, intermediate; Chemo, chemotherapy; Met, metastasis studied; GN, ganglioneuroma; GNB, ganglioneuroblastoma.

Histologic grading was assessed according to published criteria. ${ }^{15,16}$

${ }^{a}$ Cases 1 and 4 relapsed as stage 4/International Neuroblastoma Risk Group high risk. 
all eight cases. Six samples were from the time of diagnosis and two were post-chemotherapy resection samples (the specimens at time of diagnosis were similar in histology to the postchemotherapy samples but the blocks were not available.) In addition to primary tumor tissue, two cases also had lymph node metastases of stromapoor neuroblastoma available for study.

\section{In Situ Hybridization}

MYCN copy number was assessed both by chromogenic in situ hybridization ${ }^{31}$ and fluorescent in situ hybridization, evaluating separately neuroblasts from stroma-poor areas, ganglion cells from stroma-rich areas, and Schwann cells from stroma-rich areas. For fluorescent in situ hybridization, Spectrum orange- or Spectrum green-labeled bacterial artificial chromosome DNA was acquired from the Applied Centre for Genomics, Toronto, Canada (http://www.tcag.ca/) and selected according to the UCSC Genome Bioinfomatics Browser (http:// genome.ucsc.edu/, March 2006 Build). Each labeled bacterial artificial chromosome was tested on normal human lymphocyte metaphases to ensure that the bacterial artificial chromosome mapped to the correct chromosomal location. The following bacterial artificial chromosome probes were used: RP11-480N14 (MYCN), RP11-59N1 (11q23.3), RP111001I7 and RP11-1134C2 (13q14.2 and 13qtel, respectively), RP11-46G16 (17q21.1-21.2), and RP11-254I3 (18q21.1). Centromere probes for chromosomes 2, 11, and 17 were obtained from Vysis (Vysis, Abbott Molecular, Downers Grove, IL, USA). Slides for fluorescent in situ hybridization were de-waxed, pretreated with citrate buffer followed by pepsin, dehydrated, and then co-denatured with the labeled probe(s) for $10 \mathrm{~min}$ at $75^{\circ} \mathrm{C}$ using the ThermoBrite system (Abbott Molecular) and allowed to hybridize overnight at $37^{\circ} \mathrm{C}$. The slides were then processed for $2 \mathrm{~min}$ at $75^{\circ} \mathrm{C}$ in $2 \times \mathrm{SSC} /$ $0.3 \% \mathrm{NP}-40$ followed by a room-temperature wash for $1 \mathrm{~min}$ in the same buffer. The slides were then stained and mounted with DAPI/Antifade (Vectashield). Fluorescent in situ hybridization signals were visualized with an epifluorescence microscope (AxioImager Z1, Carl Zeiss Microimaging, Thornwood, NY, USA). In order to compare the fluorescent in situ hybridization results for nodular ganglioneuroblastoma involving the regions $13 q$ and 18q, 19 control cases of non-nodular ganglioneuroblastoma neuroblastoma were selected, all nonMYCN amplified. These consisted of primary tumors biopsied between 2007 and 2010, with paraffin blocks available, and included both stroma-poor and stroma-rich histologies. There were eight cases with stage 1 or 2 disease ${ }^{14}$ (low risk), two cases with stage 3 disease (intermediate risk), and nine cases of stage 4 (high risk). Normal tissue controls included the kidney, tonsil, breast, and brain.

\section{Single-Nucleotide Polymorphism Arrays}

DNA was extracted separately from neuroblastomatous and ganglioneuromatous areas of the tumors using histology from a stained section of the block as a guide. The cells in the neuroblastomatous areas were $70-95 \%$ neuroblasts, while the ganglioneuromatous areas included 5-40\% ganglionic cells with Schwann cells comprising almost all of the remaining cells. For paraffin blocks, a core from each area was removed. Formalin-fixed paraffinembedded tissue was dewaxed in xylene and rehydrated in a series of ethanol washes. Tissue was then placed in proteinase $\mathrm{K}$ buffer overnight at $37^{\circ} \mathrm{C}$, and DNA was then extracted using phenolchloroform, air dried, and reconstituted in water. A minimum of $750 \mathrm{ng}$ of DNA per sample were hybridized to the Affymetrix Human 6.0 singlenucleotide polymorphism arrays, at The Centre for Applied Genomics at The Hospital for Sick Children. The Affymetrix 6.0 single-nucleotide polymorphism array includes >906 600 single-nucleotide polymorphisms and >946 000 probes for the detection of copy number variation. DNA was digested with NspI and StyI restriction enzymes, ligated to adaptors followed by a polymerase chain reaction using primers that recognize the adaptor. PCR conditions were optimized to preferentially amplify fragments in the 200 to $1100 \mathrm{bp}$ size range. Two extra PCR cycles were done for the paraffin-embedded samples. The amplified DNA was then labeled and hybridized to a Genome-Wide Human single-nucleotide polymorphism 6.0 Array. The single-nucleotide polymorphism array data from paired samples (neuroblastoma and ganglioneuromatous components) were analyzed and integrated using the Partek Genomic Suite Software (Partek, St Louis, MO, USA). The normal reference used was the Human HapMap data set (hapmap.ncbi.nlm. nih.gov), which is derived from 270 individuals of different ethnic origins. Following the manufacturer's recommendations for copy number analysis, the minimum number of adjacent genomic markers on the array with a copy number change that would be viewed as a valid change was set at 10 (genomic segmentation parameter). Fragment lengths were restricted to $<700 \mathrm{bp}$ for the formalin-fixed paraffin-embedded samples, as previously recommended. ${ }^{32}$

\section{Results}

\section{MYCN Copy Number Gain in Neuroblasts and Ganglion Cells from Nodular Ganglioneuroblastoma}

MYCN oncogene copy number was assessed separately in neuroblasts from stroma-poor areas, ganglion cells from ganglioneuromatous areas, and Schwann cells from ganglioneuromatous areas. At least 200 nuclei were scored. Regions of neuroblastoma were $>70 \%$ neuroblasts. Ganglioneuromatous 
areas were a mixture of Schwann cells and ganglionic cells. In chromogenic in situ hybridization sections, cell types were easily identified by light microscopy. For fluorescent in situ hybridization, immediately adjacent sections stained with hematoxylin and eosin were compared with aid in identification of cell type. In ganglioneuromatous areas, ganglionic cells had larger nuceli than Schwann cells and could readily be identified by DAPI nuclear staining. Results for chromogenic in situ hybridization and fluorescent in situ hybridization were comparable, indicating that identification of cell type in fluorescent in situ hybridization sections was reliable.

$M Y C N$ copy number at the single neuroblast cell level was variable both within a given sample and between different samples from the same patient (Table 2). Some of this variation may be related to sectioning of nuclei, but differences were also seen between tumor samples, as a reflection of tumor heterogeneity. To determine whether MYCN copy number increase was related to extra copies of chromosome 2, a centromere probe for chromosome 2 was used. Seven of eight cases (all but case 6) showed 4-10 copies of MYCN, but up to 12 copies were noted in one neuroblast in case 3 (Figure 1a). Cases 1-5 had increased copies of chromosome 2; cases 6,7 , and 8 had a normal copy number for chromosome 2. Comparing the number of $M Y C N$ signals and centromere 2 signals, all cases were scored as MYCN-gained and none as MYCN amplified. ${ }^{33}$ The proportion of neuroblasts with $M Y C N$ gain varied from case to case, ranging from $10 \%$ (case 6) up to $61 \%$ (case 3 ) with the remainder in the $22-48 \%$ range. Four of the cases with $M Y C N$ gain also showed gain in ganglion cells (cases 2, 4, 5, and 8) with up to 17 copies in 1 ganglion cell in case 4 and 10-15 copies in 40\% ganglion cells in case 8 (Figure 1b), whereas the neuroblastoma component showed up to 5 copies of $M Y C N$ per neuroblast. Two other cases (1 and 6) showed normal copy number in ganglion cells and for the other two cases (3 and 7) hybridization was unsuccessful. Only 2 of 6 cases
(2 and 5) showed increased copies of chromosome 2 in the ganglion cells. Schwann cells consistently had two copies of MYCN and two copies of chromosome 2.

\section{Single-Nucleotide Polymorphism Array Analysis Comparing Neuroblastoma and Ganglioneuromatous Regions of Nodular Ganglioneuroblastoma}

There were many small regions of gain and loss that differed among cases. Although these may represent regions of importance in nodular ganglioneuroblastoma, these alterations could not readily be distinguished from background noise and, for the purposes of this study, regions scored as a definite gain or loss required a minimum size of $100 \mathrm{Mb}$ and detection in $>1$ sample. Following this approach, the neuroblastoma component of the eight cases showed gains and losses over the genome, which are summarized in Figure 2 and Table 3. The two metastatic lesions were very similar to the neuroblastoma component of their primary tumors. Changes in individual tumors varied from 1 to 25 copy number alterations. The tumors with $>10$ copy number alterations included three with unfavorable histology and two with favorable, while tumors with $<5$ copy number alterations included one with unfavorable histology and one with favorable. The most consistent alterations were gains at chromosome $1 q$ ( $4 / 8$ cases), $2 p$ ( $3 / 8$ cases), $7 p$ ( $4 / 8$ cases), $7 q$ (6/8 cases), 12q (5/8 cases), 13q (5/8 cases), 17q ( $7 / 8$ cases), and $18 q$ ( $4 / 8$ cases), and losses at chromosome $11 q$ (5/8 cases) and $14 q$ (5/8 cases). There was no correlation between the copy number alteration and histology (favorable vs unfavorable), or with patient stage, risk category, or outcome.

The results from the ganglioneuromatous components of each case are summarized in Figure 3. In contrast to the neuroblastomatous component, fewer copy number alterations were detected in the ganglioneuromatous component, ranging from 0 to 3 , except for case 5 that showed 7. In general, a copy number alteration identified in the ganglioneuromatous component was also detected in the

Table 2 Result of in situ hybridization for MYCN

\begin{tabular}{|c|c|c|c|c|c|c|}
\hline \multirow[b]{2}{*}{ Case } & \multicolumn{3}{|c|}{ NB component } & \multicolumn{3}{|c|}{ GNB component } \\
\hline & $\begin{array}{l}\text { Cells with } \geq 3 \text { copies } \\
\text { of centromere } 2\end{array}$ & $\begin{array}{c}\text { Cells with } \geq 3 \text { copies } \\
\text { of } M Y C N\end{array}$ & $\begin{array}{c}\text { Maximum } \\
\text { MYCN copy \# }\end{array}$ & $\begin{array}{l}\text { Cells with } \geq 3 \text { copies } \\
\text { of centromere } 2\end{array}$ & $\begin{array}{c}\text { Cells with } \geq 3 \text { copies } \\
\text { of MYCN }\end{array}$ & $\begin{array}{l}\text { Maximum } \\
\text { MYCN copy }\end{array}$ \\
\hline 1 & $24 \%$ & $37 \%$ & 10 & $0 \%$ & $20 \%$ & 4 \\
\hline 2 & $68 \%$ & $48 \%$ & 7 & $21 \%$ & $25 \%$ & 7 \\
\hline 3 & $65 \%$ & $61 \%$ & 12 & $0 \%$ & Failed & Failed \\
\hline 4 & $27 \%$ & $22 \%$ & 7 & Failed & $24 \%$ & 17 \\
\hline 5 & $30 \%$ & $30 \%$ & 5 & $23 \%$ & $38 \%$ & 6 \\
\hline 6 & $0 \%$ & $10 \%$ & 4 & $0 \%$ & $16 \%$ & 4 \\
\hline 7 & $0 \%$ & $26 \%$ & 5 & Failed & Failed & Failed \\
\hline 8 & $0 \%$ & $30 \%$ & 5 & $0 \%$ & $80 \%$ & 15 \\
\hline
\end{tabular}

Abbreviations: NB, neuroblastomatous component of nodular ganglioneuroblastoma; GN, ganglioneuromatous component of nodular ganglioneuroblastoma. 

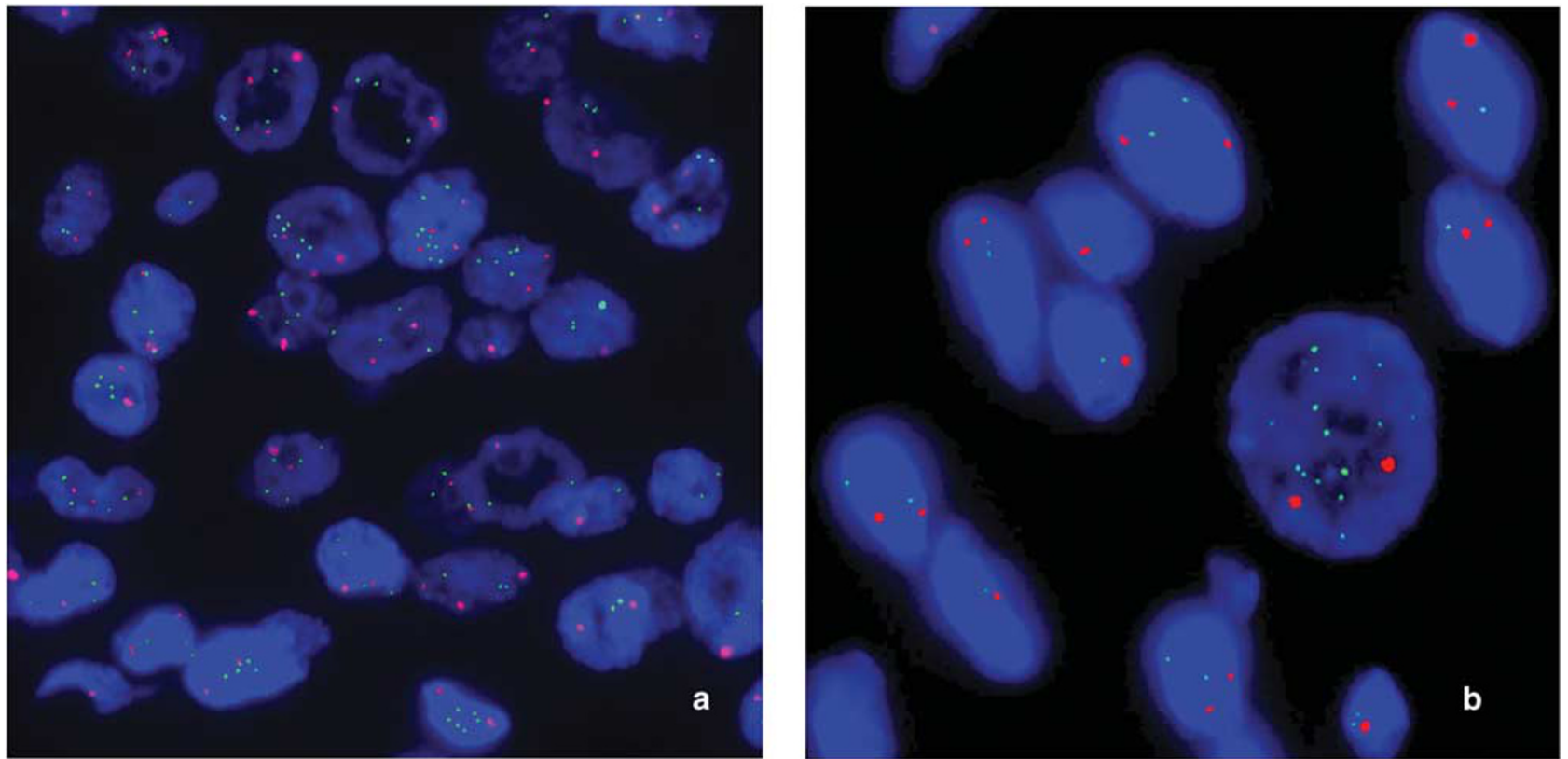

Figure 1 Fluorescent in situ hybridization for $M Y C N$ in nodular ganglioneuroblastoma. MYCN gain (green probe) compared with a centromeric probe for chromosome 2 (red probe) in the neuroblastomatous component of case 3 (a) and the ganglioneuromatous component of case $8(\mathbf{b})$. In the latter, the gain is restricted to the large ganglion cell in the centre, while adjacent Schwann cells show a normal MYCN copy number.
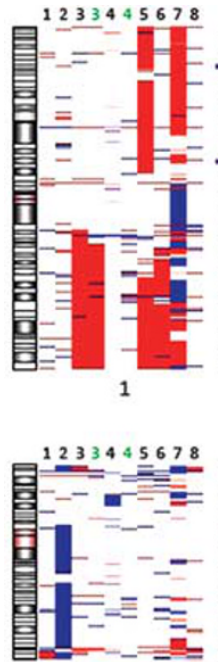

9

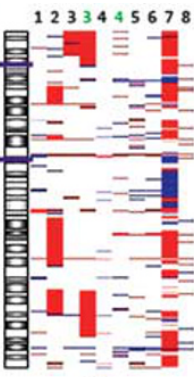

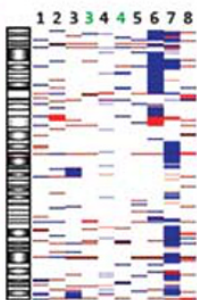

3

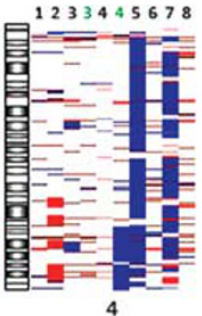

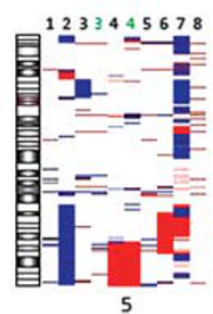

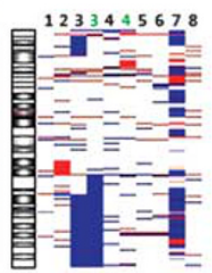

6
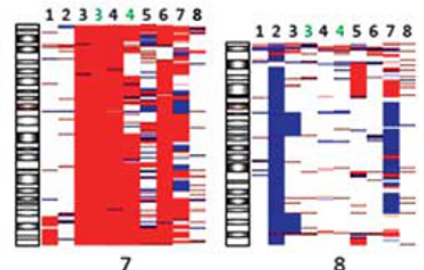

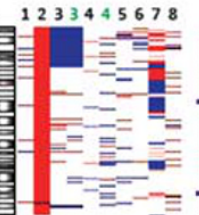

10

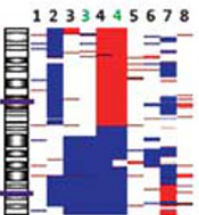

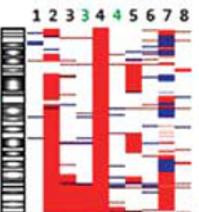

12

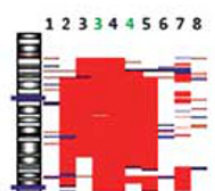

13

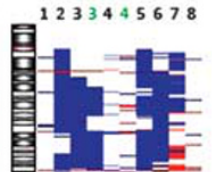

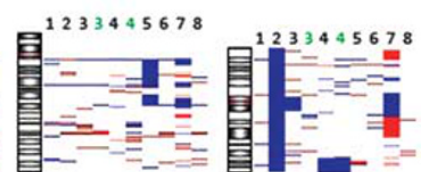

15

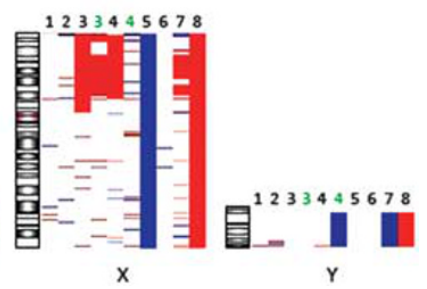

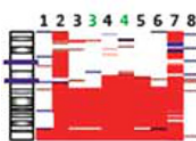

17

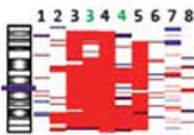

18

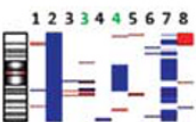

19

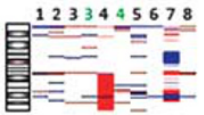

20

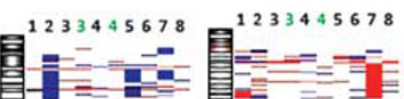

21
22

Figure 2 Compilation of single-nucleotide polymorphism array results for the neuroblastoma component of the eight cases (1-8) of nodular ganglioneuroblastoma based on DNA extracted from formalin-fixed tissue. Two cases had metastases of neuroblastoma type tumor analyzed as well (green 3 and 4). Gains are indicated in red and losses in blue. The results are summarized in Table 3. The locations of the fluorescent in situ hybridization probes used in the study are marked with a purple bar on the respective chromosomes. 
Table 3 Summary of chromosomal gains and losses in nodular ganglioneuroblastoma cases detected in single nucleotide polymorphism array

\begin{tabular}{|c|c|c|c|c|c|c|c|c|c|c|c|c|c|c|}
\hline Case & $1 N B$ & $2 N B$ & $3 N B$ & 3MET & $3 G N$ & $4 N B$ & $4 M E T$ & $4 G N$ & $5 N B$ & $5 G N$ & $6 N B$ & $7 N B$ & $8 N B$ & $8 G N$ \\
\hline HISTO & $\mathrm{U}$ & $\mathrm{U}$ & $\mathrm{U}$ & & & $\mathrm{U}$ & & & $\mathrm{F}$ & & $\mathrm{F}$ & $\mathrm{F}$ & $\mathrm{F}$ & \\
\hline 1p gain & & & & & & & & & $\mathrm{X}$ & $\mathrm{X}$ & & $\mathrm{X}$ & & \\
\hline $1 \mathrm{q}$ gain & & & $\mathrm{X}$ & $\mathrm{X}$ & & & & & $\mathrm{X}$ & & $\mathrm{X}$ & $\mathrm{X}$ & & \\
\hline $2 p$ gain & & $\mathrm{X}$ & $\mathrm{X}$ & $\mathrm{X}$ & & & & & & $\mathrm{X}$ & & $\mathrm{X}$ & & \\
\hline $2 q$ gain & & $\mathrm{X}$ & & $\mathrm{X}$ & & & & & & & & $\mathrm{X}$ & & \\
\hline $3 p$ loss & & & & & & & & & & & $\mathrm{X}$ & $\mathrm{X}$ & & \\
\hline $4 p$ loss & & & & & & & & & $\mathrm{X}$ & $\mathrm{X}$ & & $\mathrm{X}$ & & \\
\hline $4 q$ loss & & & & & & & $\mathrm{X}$ & & $\mathrm{X}$ & & & $\mathrm{X}$ & & \\
\hline $5 \mathrm{p}$ loss & & & $\mathrm{X}$ & & & & & & & $\mathrm{X}$ & & $\mathrm{X}$ & & \\
\hline $5 q$ gain & & & & & & $\mathrm{X}$ & $\mathrm{X}$ & & & & $\mathrm{X}$ & $X$ & & \\
\hline $6 \mathrm{q}$ loss & & & $\mathrm{X}$ & $\mathrm{X}$ & & & & & & & & $\mathrm{X}$ & & \\
\hline $7 p$ gain & & & $\mathrm{X}$ & $\mathrm{X}$ & $\mathrm{X}$ & $\mathrm{X}$ & $\mathrm{X}$ & & & & $\mathrm{X}$ & $\mathrm{X}$ & & \\
\hline $7 q$ gain & $\mathrm{X}$ & & $\mathrm{X}$ & $\mathrm{X}$ & $\mathrm{X}$ & $\mathrm{X}$ & $\mathrm{X}$ & & $\mathrm{X}$ & & $\mathrm{X}$ & $\mathrm{X}$ & & \\
\hline 8p gain & & & & & & & & & $\mathrm{X}$ & & & $\mathrm{X}$ & & \\
\hline $8 \mathrm{q}$ loss & & $\mathrm{X}$ & $\mathrm{X}$ & & & & & & & & & $\mathrm{X}$ & & \\
\hline 10p gain & & $\mathrm{X}$ & & & & & & & & & & $\mathrm{X}$ & & \\
\hline $10 \mathrm{q}$ loss & & & & & & & & & & $\mathrm{X}$ & & $\mathrm{X}$ & & \\
\hline $11 p$ loss & & $\mathrm{X}$ & & & & & & & & & & $X$ & & \\
\hline 11q loss & & $\mathrm{X}$ & $\mathrm{X}$ & $\mathrm{X}$ & & $\mathrm{X}$ & $\mathrm{X}$ & & & & $\mathrm{X}$ & $\mathrm{X}$ & $\mathrm{O}$ & $\mathrm{O}$ \\
\hline $12 p$ gain & & $\mathrm{X}$ & & & & $\mathrm{X}$ & & & $\mathrm{X}$ & & & & & \\
\hline 12q gain & & $X$ & $X$ & $\mathrm{X}$ & $\mathrm{X}$ & $\mathrm{X}$ & & & $\mathrm{X}$ & & & $\mathrm{X}$ & & \\
\hline 13q gain & $\mathrm{O}$ & $\mathrm{X}$ & $\mathrm{X}$ & $\mathrm{X}$ & & $\mathrm{X}$ & $\mathrm{X}$ & $\mathrm{X}$ & $\mathrm{X}$ & & & $\mathrm{X}$ & $\mathrm{O}$ & \\
\hline $14 \mathrm{q}$ loss & & $\mathrm{X}$ & $\mathrm{X}$ & $\mathrm{X}$ & & & & & $\mathrm{X}$ & & $\mathrm{X}$ & $\mathrm{X}$ & & \\
\hline $16 q$ loss & & $X$ & & & & $\mathrm{X}$ & $\mathrm{X}$ & & & & & $\mathrm{X}$ & & \\
\hline $17 p$ gain & & $\mathrm{X}$ & & & & & & & & & & $\mathrm{X}$ & & \\
\hline $17 q$ gain & $\mathrm{X}$ & $X$ & $\mathrm{X}$ & $\mathrm{X}$ & & $\mathrm{X}$ & $\mathrm{X}$ & $\mathrm{X}$ & $\mathrm{X}$ & $\mathrm{X}$ & $\mathrm{X}$ & $\mathrm{X}$ & $\mathrm{O}$ & \\
\hline 18p gain & & & $\mathrm{X}$ & $X$ & & $\mathrm{X}$ & & & $\mathrm{X}$ & & & & & \\
\hline $18 \mathrm{q}$ gain & $\mathrm{O}$ & $\mathrm{X}$ & $\mathrm{X}$ & $\mathrm{X}$ & & $\mathrm{X}$ & $\mathrm{X}$ & & $\mathrm{X}$ & & $\mathrm{O}$ & & & \\
\hline $19 p$ gain & & & & & & & & & & $\mathrm{X}$ & & & $\mathrm{X}$ & $\mathrm{X}$ \\
\hline 19p loss & & $\mathrm{X}$ & & & & & & & & & & $\mathrm{X}$ & & \\
\hline $19 q$ loss & & $\mathrm{X}$ & & & & & $\mathrm{X}$ & & & & & & & \\
\hline 21q loss & & $\mathrm{X}$ & & & & & & & $\mathrm{X}$ & & & & & \\
\hline TOTAL & 2 & 17 & 14 & 13 & 3 & 11 & 10 & 2 & 14 & 7 & 8 & 25 & 1 & 1 \\
\hline
\end{tabular}

Abbreviations: HISTO, histology; U, unfavorable; F, favorable; NB, neuroblastomatous component of nodular ganglioneuroblastoma; MET, metastasis; GN, ganglioneuromatous component of nodular ganglioneuroblastoma; X, detected by single nucleotide polymorphism array; $\mathrm{O}$, detected by fluorescent in situ hybridization only.

neuroblastoma component from the same tumor, although for some tumors the neuroblastomatous component showed additional alternations not detected in the ganglioneuromatous component. The one exception was case 5 in which gains at $2 p$ and 19p, and losses at 5p and 10q, were found in the ganglioneuromatous component but not in the neuroblastomatous component.

\section{Fluorescent In Situ Hybridization Validation of Single-Nucleotide Polymorphism Array Results at the Cellular Level}

As gains may be underestimated and losses overestimated in our analysis based on paraffinembedded tissue, fluorescent in situ hybridization was performed to confirm the single-nucleotide polymorphism array results, and to determine which cell types showed the genetic changes that were detected. Fluorescent in situ hybridization was performed for chromosome regions $11 \mathrm{q}, 13 \mathrm{q}$, $17 q$, and 18q. All the neuroblastic samples with abnormalities in these chromosomes detected by single-nucleotide polymorphism array showed the same changes by fluorescent in situ hybridization (Figure 4a). Of note, fluorescent in situ hybridization identified changes that were missed by singlenucleotide polymorphism array analysis, including gains at 13q (two cases), 17q (one case) and 18q (two cases), and loss at 11q (1 case). Thus, chromosome $13 q$ gain was detected in seven/eight cases, chromosome $17 q$ gain in eight/eight cases, chromosome 18 gain in six/eight cases and chromosome 11q loss in five/eight cases.

Fluorescent in situ hybridization results for the ganglioneuromatous component were scored separately for ganglion cells and Schwann cells (Figure 4b). Hybridization studies for the ganglioneuromatous portion of case 7 failed; for the other seven cases, studies were successful. In contrast, single-nucleotide polymorphism array results were only successful for the ganglioneuromatous component in four cases, due to limited material. All changes detected in ganglion cells were also found in the neuroblastoma cells for the same case. Gains were detected in 

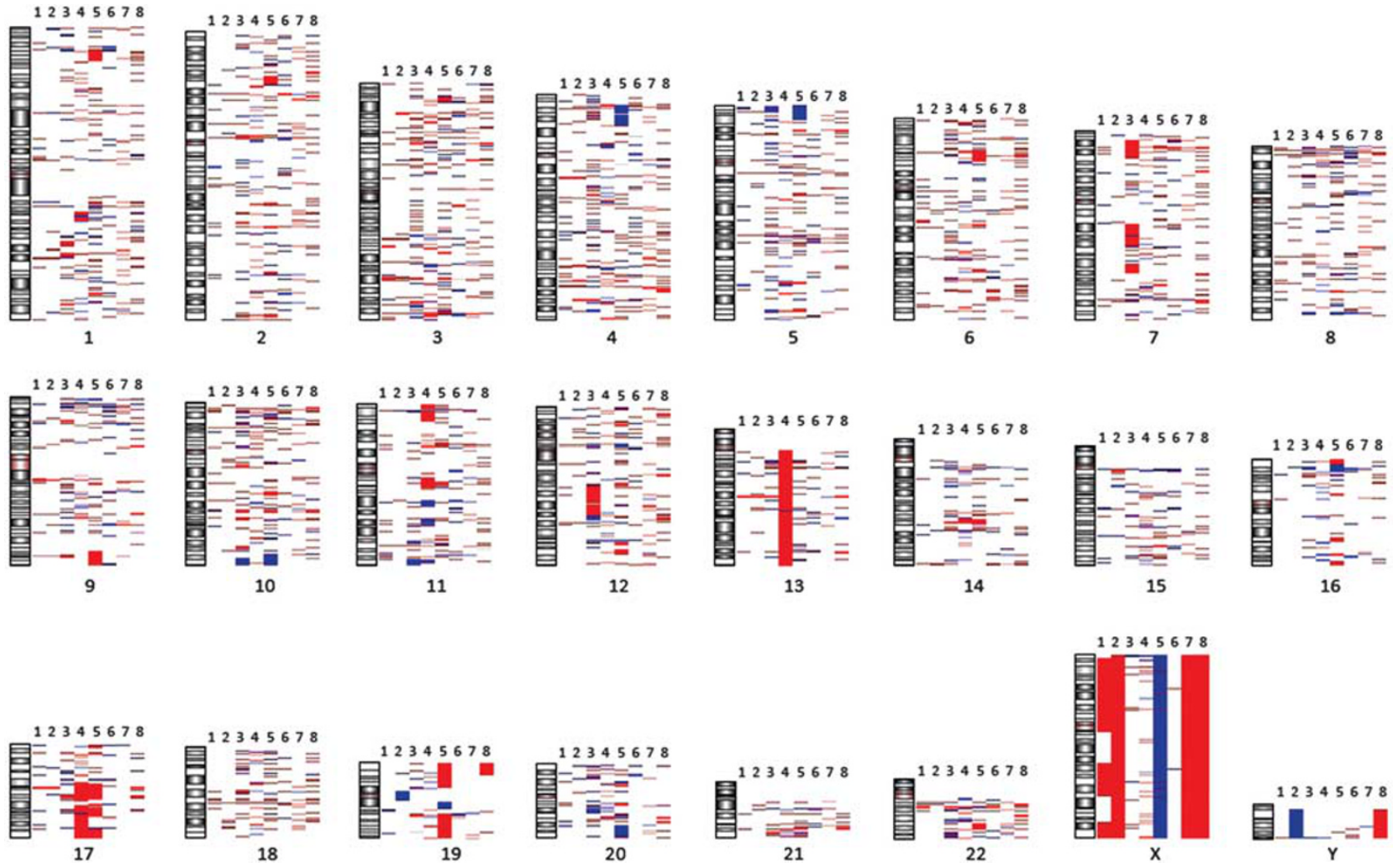

Figure 3 Compilation of single-nucleotide polymorphism array results for the ganglioneuromatous component of the eight cases of nodular ganglioneuroblastoma based on DNA extracted from formalin-fixed tissue. Gains are indicated in red and losses in blue. The results are included in Table 3 .
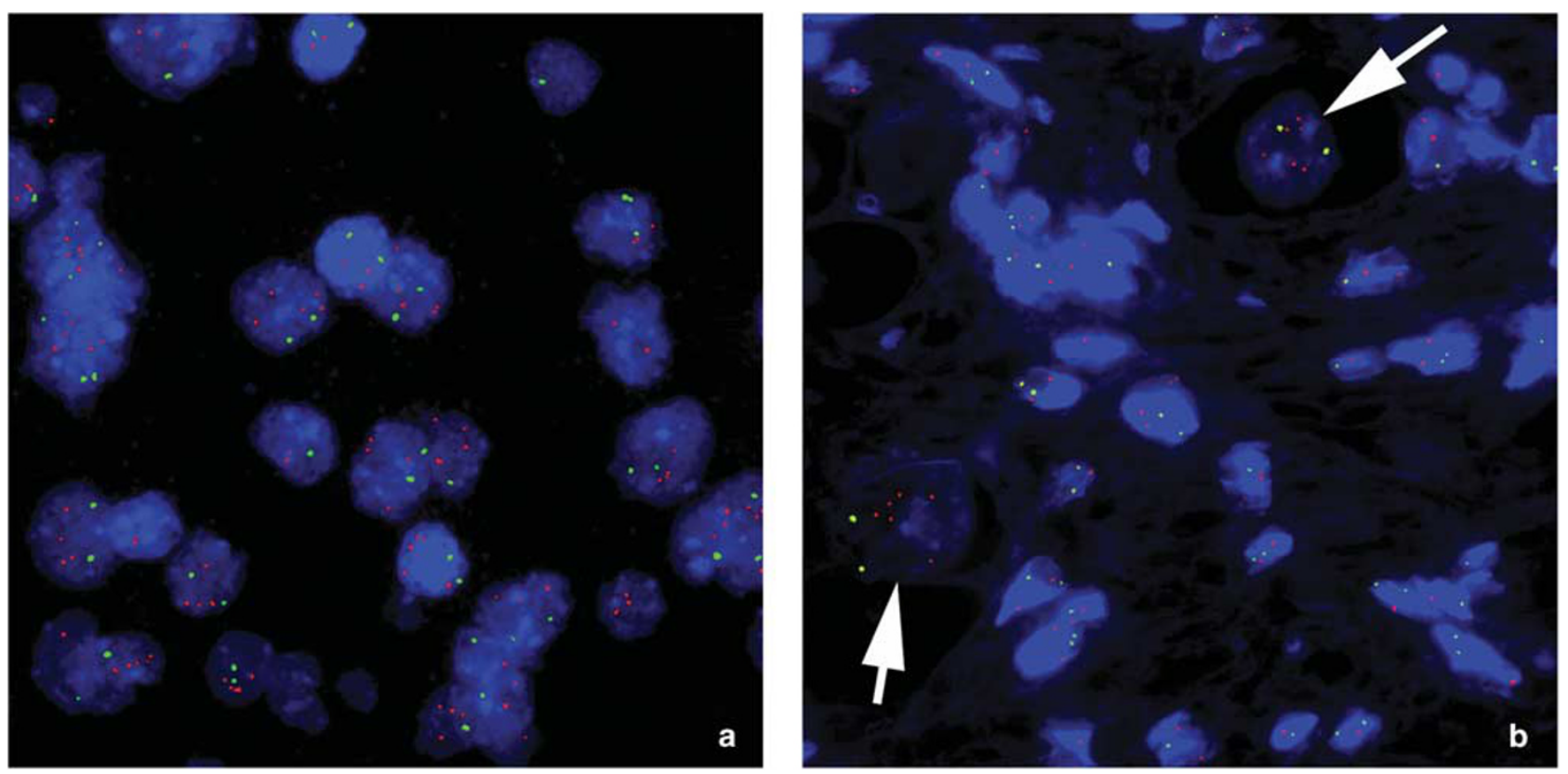

Figure 4 Fluorescent in situ hybridization for 17q in nodular ganglioneuroblastoma. Case showing 17q gain (red probe) compared with a centromere probe for chromosome 17 (green) in the neuroblastomatous component (a) and the ganglioneuromatous component (b). In the latter, the gain is restricted to the larger ganglion cells (arrows), while adjacent Schwann cells show a normal 17q copy number. 
ganglion cells at chromosome 13q (six/six cases), chromosome 17q (three/six cases), chromosome 18 (five/five cases), and loss at chromosome 11q (one/six cases). Unlike MYCN, the copy numbers for regions of gain were comparable in the neuroblasts and ganglion cells for each individual tumor. Schwann cells were consistently diploid for all probes.

\section{Fluorescent In Situ Hybridization Results for 13q and 18q for Non-Nodular Ganglioneuroblastoma Neuroblastoma}

Non-nodular ganglioneuroblastoma cases of neuroblastoma were studied to determine whether gain of $13 q$ and $18 q$ was specific for the nodular ganglioneuroblastoma subtype. Only non-MYCN-amplified tumors were included, as the nodular ganglioneuroblastoma cases in our series were not amplified, which is in keeping with previous reports. ${ }^{13}$ Of the 19 cases analyzed, hybridization was successful in 18 cases for 13q and in 16 cases for 18q; at least 1 of the 2 probes worked in every case. For 13q, a gain was detected in six/eight low-risk cases, two/two intermediate-risk cases, and five/eight high-risk cases. For 18q, a gain was detected in five/six low-risk cases, two/two intermediate-risk cases, and eight/ eight high-risk cases. All normal tissues (kidney, brain, tonsil, and breast) analyzed had a normal copy number for $13 q$ and $18 q$.

\section{Discussion}

Neuroblastoma is characterized by multiple chromosomal gains and losses (reviewed in refs 1,3), but whether these are detected specifically in the rare subtype of nodular ganglioneuroblastoma is not well known. It has been hypothesized that the neuroblastoma and ganglioneuromatous components are separate clones. ${ }^{11,16}$ The ganglioneuromatous component is proposed to be a biologically less aggressive clone, whereas the neuroblastoma component is proposed to be clone that determines the clinical behavior of the tumor. The current system of deriving the histologic prognosis in nodular ganglioneuroblastoma based on the neuroblastoma component alone ${ }^{15,16}$ reflects acceptance of the existence of separate clones in nodular ganglioneuroblastoma. However, there are few genetic data to support this concept. The main purpose of our study was to investigate whether there are distinct copy number changes to support separate clonal origins for the neuroblastoma and ganglioneuromatous components,

One study reporting a genetic difference between the neuroblastoma and ganglioneuromatous components demonstrated MYCN gene amplification in the ganglioneuromatous component of one tumor but not in the neuroblastoma component. ${ }^{34}$ The analysis was done by Southern blotting that pools DNA and it is not possible to know what proportion of cells in the pool are actually tumor cells. Moreover, a pretreatment biopsy in the same patient identified $M Y C N$ amplification in the neuroblastoma component. In general, for neuroblastoma, $>10$ copies of the MYCN gene is a poor prognostic indicator that occurs in $\sim 20 \%$ of neuroblastoma tumors but more commonly $(\sim 40 \%)$ in high-stage tumors (stage 3 and 4) and uncommonly (5-10\%) in low-stage tumors (stage 1, 2, or $4 S$ ). ${ }^{3,6,8,35,36}$ In contrast, $M Y C N$ amplification is uncommon in nodular ganglioneuroblastoma regardless of outcome, occurring in only about $2 \%$ of cases. ${ }^{13,19,37}$

Given that nodular ganglioneuroblastoma is a heterogeneous tumor by definition, we first asked whether MYCN amplification could have been underestimated in previous studies, in which cases were analyzed by techniques based on pooled extracted DNA such as Southern blotting or PCR, or by fluorescent in situ hybridization, in either case not taking into account the Schwann cell component of nodular ganglioneuroblastoma. Thus, we determined MYCN copy number in individual cells, assessing neuroblasts, ganglion cells, and Schwann cells separately. No cases in our study showed MYCN amplification; however, we frequently detected $M Y C N$ gain in the neuroblastomatous component as well as in the ganglion cells of the ganglioneuromatous component, but not the Schwann cells. In the majority of cases, MYCN gain was due to increased copies of chromosome 2. There were two cases in which the MYCN copy number was higher in some $(<5 \%)$ ganglion cells than in neuroblasts. This basis for this finding is unclear, but we speculate this might result from failure of double minute chromosomes (containg $M Y C N$ ) to divide evenly during mitosis, leading to accumulation in a subset of ganglion cells, which then no longer divide.

Many different regions of gain and loss have been reported in neuroblastoma, but nodular ganglioneuroblastoma has been infrequently studied and specific changes have not been identified. ${ }^{28,30}$ We next examined other regions of the genome for gains and losses using single-nucleotide polymorphism arrays to identify copy number alterations that might be specific to the nodular ganglioneuroblastoma subtype. One potential limitation of our study was that two of the eight cases were post chemotherapy, and chemotherapy might introduce additional genetic changes. However, no gains or losses were seen in these two cases that were not also seen in at least one case that had not been exposed to chemotherapy, and it was therefore felt reasonable to include these two cases.

The neuroblastomatous (stroma-poor) component showed losses at $11 \mathrm{q}$ and $14 \mathrm{q}$, and gains at $1 \mathrm{q}, 2 \mathrm{p}$, $7 p, 7 q, 12 q, 13 q, 17 q$, and 18q, many of which have been reported previously in neuroblastoma. Loss of $11 \mathrm{q}$ and $14 \mathrm{q}$, and gain of $17 \mathrm{q}$ are well-established poor prognostic indicators in neuroblastoma (reviewed in ref. 3). Other copy number alterations 
detected in our study have not commonly been identified as 'regions of interest' in neuroblastoma, such as gains at $13 \mathrm{q}$ and 18q. Thus, we asked whether either of these chromosomal regions might provide a genomic signature change specific to nodular ganglioneuroblastoma; however, we detected similar gains in the majority of non-nodular ganglioneuroblastoma cases that we tested. Moreover, these regions of gain have also been reported in other array studies on neuroblastoma. ${ }^{23,24,28-30,38}$ Thus, although our results suggest there may be a genetic profile characteristic of nodular ganglioneuroblastoma, it would not appear to distinguish this subtype of neuroblastoma from others. In our series, there was no correlation between the total number of copy number alterations or specific gains and losses and histologic prognostic categories (favorable vs unfavorable) or patient outcome. It is possible that smaller copy number alterations might be significant clinically, but such changes would be beyond the technical limitations of our study that was based on formalin-fixed paraffin-embedded samples.

One unique aspect of our study was the ability to separately analyze the genomic pattern separately for the neuroblastomatous (stroma-poor) and ganglioneuromatous components of nodular ganglioneuroblastoma. The ganglioneuromatous component of nodular ganglioneuroblastoma generally showed far fewer gains and losses by single-nucleotide polymorphism arrays than those detected in the neuroblastoma component. No case showed all of the same changes as the neuroblastoma component and two showed no copy alterations. At first, these results might suggest that the ganglioneuromatous and neuroblastomatous components of nodular ganglioneuroblastoma are genetically distinct, providing support for the concept that they are separate clones; however, fluorescent in situ hybridization studies established that the same gains and losses were present in the ganglion cells in the ganglioneuromatous component, whereas the Schwann cells were always normal (diploid) for these regions. As the majority of the cells in the ganglioneuromatous component are Schwann cells, the gains and losses in ganglion cells would be diluted out in the pooled DNA used for single-nucleotide polymorphism array, accounting for why so few genetic changes were found in our study in ganglioneuromatous component. The same effect is more evident in the copy number studies for MYCN. By single-nucleotide polymorphism array, only two cases show a gain at 2 p24, whereas all cases showed a gain of MYCN by fluorescent in situ hybridization. In the ganglioneuromatous component, some ganglion cells showed a gain by fluorescent in situ hybridization as well, but no tumor showed a gain of chromosome 2 p24 on single-nucleotide polymorphism array. The gain of $M Y C N$ was seen only in a subset of neuroblasts (generally $20-30 \%$ ) and occcasional ganglion cells, and these cells were diluted out by the cells without MYCN gain, and not detected by single-nucleotide polymorphism array. This dilutional effect has received little attention in previous studies. Our findings underscore the need to perform fluorescent in situ hybridization studies in addition to array studies, otherwise specific regions of gain or loss may be missed.

Our finding that the Schwann cells were always diploid by fluorescent in situ hybridization indicates these cells have a different origin from the neuroblastoma and ganglion cells, and likely lack malignant potential. Our results give support to the view that the Schwann cells in neuroblastoma are reactive cells rather than neoplastic, a concept that is controversial and supported by some reports ${ }^{39-41}$ but refuted by others. ${ }^{30,42-44}$ Results interpreted as supporting this concept are based on the detection of one or more genetic abnormalities in neuroblasts, whereas Schwann cells from the same tumor are normal for the same genetic region(s). Other studies have refuted this idea by demonstrating genetic abnormalities that are interpreted to reside in Schwann cells, concluding these cells are neoplastic. However, these studies have generally been based on extracted DNA from ganglioneuromatous regions of neuroblastoma. ${ }^{30,42-44}$ We have shown in our study that the ganglioneuromatous component of nodular ganglioneuroblastoma contains ganglion cells that share the same genetic abnormalities as neuroblasts and such abnormalities can sometimes be detected on arrays and do not necessarily imply the changes are present within Schwann cells. In fact, when fluorescent in situ hybridization was then performed in one of these studies, ${ }^{44}$ no abnormalities were detected in Schwann cells.

The combined results of single-nucleotide polymorphism array and fluorescent in situ hybridization, applied separately to the neuroblastomatous and ganglioneuromatous components, lead us to the conclude that in the case of nodular ganglioneuroblastoma, the ganglion cells and neuroblastoma cells share genetic changes, and therefore may be clonally related. This conclusion would appear to be in conflict with the concept that the ganglioneuromatous and neuroblastomatous components of nodular ganglioneuroblastoma are derived from separate clones, with the neuroblastoma clone the one that determines the biology of the tumour, and therefore the component that is used for histologic prognostic grading. ${ }^{11,16}$ It may be more appropriate to regard the neuroblastoma component as representative of the biology of the entire tumor, but with specific histologic findings that can be graded in a meaningful prognostic way, whereas the ganglioneuromatous component cannot be. It is still possible that neuroblasts and ganglion cells in nodular ganglioneuroblastoma represent subclones, differing from each other at a genetic level that was beyond the limits used in this study. For example, case 5 showed gains and losses in the ganglioneuromatous component that were not detected in the neuroblastomatous component. Whether these are true clonal 
differences or variations introduced by the use of formalin-fixed material is not clear. Even though neuroblasts and ganglion cells share the same signal changes by fluorescent in situ hybridization, this could still indicate different changes in terms of the amount of genetic material gained or lost. Such additional genetic changes could determine distinct biological properties of these cells. Our results do not provide insight into whether the ganglionic cells in nodular ganglioneuroblastoma are derived from neuroblasts or whether neuroblasts in nodular ganglioneuroblastoma might be derived from dedifferentiated ganglion cells, only that these two cell types appear to be clonally related. Moreover, our study is limited by the small number of samples studied, a reflection of the rarity of this neuroblastoma subtype. As such, our results should be considered as preliminary in nature and needing verification by the study of additional cases. Further studies comparing the cell types in both cases with favorable and unfavorable outcomes may identify critical genetic changes that explain the biology of nodular ganglioneuroblastoma.

\section{Acknowledgments}

This work was funded by the James Fund for Neuroblastoma Research, Hospital for Sick Children

\section{Disclosure/conflict of interest}

The authors declare no conflict of interest.

\section{References}

1 Maris JM. Recent advances in neuroblastoma. N Engl J Med 2010;362:2202-2211.

2 London WB, Castleberry RP, Matthay KK, et al. Evidence for an age cutoff greater than 365 days for neuroblastoma risk group stratification in the Children's Oncology Group. J Clin Oncol 2005;23: 6459-6465.

3 Maris J, Hogarty M, Bagatell R, et al. Neuroblastoma. Lancet 2007;369:2106-2120.

4 Riley R, Heney D, Jones D, et al. A systematic review of molecular and biological tumor markers in neuroblastoma. Clin Cancer Res 2004;10:4-12.

5 Shimada H, Chatten J, Newton WJ, et al. Histopathologic prognostic factors in neuroblastic tumors: definition of subtypes of ganglioneuroblastoma and an age-linked classification of neuroblastomas. J Natl Cancer Inst 1984;73:405-416.

6 Brodeur G, Seeger R, Schwab M, et al. Amplification of $\mathrm{N}$-myc in untreated human neuroblastomas correlates with advanced disease stage. Science 1984;224: 1121-1124.

7 Schwab M, Alitalo K, Klempnauer K, et al. Amplified DNA with limited homology to myc cellular oncogene is shared by human neuroblastoma cell lines and a neuroblastoma tumor. Nature 1983;305:245-248.
8 Seeger R, Brodeur G, Sather $\mathrm{H}$, et al. Association of multiple copies of the $\mathrm{N}$-myc oncogene with rapid progession of neuroblastomas. N Engl J Med 1985; 313:1111-1116.

9 Baker DL, Schmidt ML, Cohn SL, et al. Outcome after reduced chemotherapy for intermediate-risk neuroblastoma. N Engl J Med 2010;363:1313-1323.

10 Joshi V. Peripheral neuroblastic tumors: pathologic classification based on recommendations of international neuroblastoma pathology committee (Modification of Shimada classification). Pediatr Dev Pathol 2000;3:184-199.

11 Shimada H, Ambros IM, Dehner LP, et al. The International Neuroblastoma Pathology Classification (the Shimada system). Cancer 1999;86:364-372.

12 Shimada H, Umehara S, Monobe Y, et al. International neuroblastoma pathology classification for prognostic evaluation of patients with peripheral neuroblastic tumors: a report from the Children's Cancer Group. Cancer 2001;92:2451-2461.

13 Angelini P, London WB, Cohn SL, et al. Characteristics and outcome of patients with ganglioneuroblastoma, nodular subtype: a report from the INRG project. Eur J Cancer 2012;48:1185-1191.

14 Cohn SL, Pearson AD, London WB, et al. The International Neuroblastoma Risk Group (INRG) classification system: an INRG Task Force report. J Clin Oncol 2009;27:289-297.

15 Peuchmaur M, d'Amore ES, Joshi VV, et al. Revision of the International Neuroblastoma Pathology Classification: confirmation of favorable and unfavorable prognostic subsets in ganglioneuroblastoma, nodular. Cancer 2003;98:2274-2281.

16 Umehara S, Nakagawa A, Matthay KK, et al. Histopathology defines prognostic subsets of ganglioneuroblastoma, nodular. Cancer 2000;89:1150-1161.

17 Ikeda H, Iehara T, Tsuchida Y, et al. Experience with International Neuroblastoma Staging System and Pathology Classification. Br J Cancer 2002;86: 1110-1116.

18 Navarro S, Amann G, Beiske K, et al. Prognostic value of International Neuroblastoma Pathology Classification in localized resectable peripheral neuroblastic tumors: a histopathologic study of localized neuroblastoma European Study Group 94.01 Trial and Protocol. J Clin Oncol 2006;24:695-699.

19 Okamatsu C, London WB, Naranjo A, et al. Clinicopathological characteristics of ganglioneuroma and ganglioneuroblastoma: a report from the CCG and COG. Pediatr Blood Cancer 2009;53:563-569.

20 Chen Q, Bilke S, Wei J, et al. cDNA array-CGH profiling identifies genomic alterations specific to stage and MYCN-amplification in neuroblastoma. BMC Genomics 2004;5:70.

21 Janoueix-Lerosey I, Schleiermacher G, Michels E, et al. Overall genomic pattern is a predictor of outcome in neuroblastoma. J Clin Oncol 2009;27:1026-1033.

22 Mosse YP, Diskin SJ, Wasserman N, et al. Neuroblastomas have distinct genomic DNA profiles that predict clinical phenotype and regional gene expression. Genes Chromosomes Cancer 2007;46: 936-949.

23 Scaruffi P, Coco S, Cifuentes F, et al. Identification and characterization of DNA imbalances in neuroblastoma by high-resolution oligonucleotide array comparative genomic hybridization. Cancer Genet Cytogenet 2007; $17: 20-29$ 
24 Spitz R, Oberthuer A, Zapatka M, et al. Oligonucleotide array-based comparative genomic hybridization (aCGH) of 90 neuroblastomas reveals aberration patterns closely associated with relapse pattern and outcome. Genes Chromosomes Cancer 2006;45:1130-1142.

25 Vandesompele J, Baudis M, De Preter K, et al. Unequivocal delineation of clinicogenetic subgroups and development of a new model for improved outcome prediction in neuroblastoma. J Clin Oncol 2005;23: 2280-2299.

26 Caren H, Erichsen J, Olsson L, et al. High-resolution array copy number analyses for detection of deletion, gain, amplification and copy-neutral $\mathrm{LOH}$ in primary neuroblastoma tumors: four cases of homozygous deletions of the CDKN2A gene. BMC Genomics 2008;9:353.

27 Fix A, Lucchesi C, Ribeiro A, et al. Characterization of amplicons in neuroblastoma: high-resolution mapping using DNA microarrays, relationship with outcome, and identification of overexpressed genes. Genes Chromosomes Cancer 2008;47:819-834.

28 McArdle L, McDermott M, Purcell R, et al. Oligonucleotide microarray analysis of gene expression in neuroblastoma displaying loss of chromosome 11q. Carcinogenesis 2004;25:1599-1609.

29 Toraman AD, Keser I, Luleci G, et al. Comparative genomic hybridization in ganglioneuroblastomas. Cancer Genet Cytogenet 2002;132:36-40.

30 Bourdeaut F, Ribeiro A, Paris R, et al. In neuroblastic tumours, Schwann cells do not harbour the genetic alterations of neuroblasts but may nevertheless share the same clonal origin. Oncogene 2008;27:3066-3071.

31 Thorner P, Ho M, Chilton-MacNeill S, et al. Use of chromogenic in situ hybridization to identify MYCN gene copy number in neuroblastoma using routine tissue sections. Am J Surg Pathol 2006;30:635-642.

32 Lyons-Weiler M, Hagenkord J, Sciulli C, et al. Optimization of the Affymetrix GeneChip Mapping $10 \mathrm{~K} 2.0$ Assay for routine clinical use on formalin-fixed paraffinembedded tissues. Diagn Mol Pathol 2008;17:3-13.

33 Ambros PF, Ambros IM, Brodeur GM, et al. International consensus for neuroblastoma molecular diagnostics: report from the International Neuroblastoma Risk Group (INRG) Biology Committee. Br J Cancer 2009;100:1471-1482.
34 Schmidt ML, Salwen HR, Chagnovich D, et al. Evidence for molecular heterogeneity in human ganglioneuroblastoma. Pediatr Pathol 1993;13:787-796.

35 Katzenstein H, Bowman L, Brodeur G, et al. The prognostic significance of age, MYCN oncogene amplification, tumor cell ploidy, and histology in 110 infants with stage $D(S)$ neuroblastoma: The Pediatric Oncology Group experience. J Clin Oncol 1998;16: 2007-2017.

36 Bagatell R, Beck-Popovic M, London WB, et al. Significance of MYCN amplification in international neuroblastoma staging system stage 1 and 2 neuroblastoma: a report from the International Neuroblastoma Risk Group database. J Clin Oncol 2009;27:365-370.

37 Goto S, Umehara S, Gerbing RB, et al. Histopathology (International Neuroblastoma Pathology Classification) and MYCN status in patients with peripheral neuroblastic tumors: a report from the Children's Cancer Group. Cancer 2001;92:2699-2708.

38 Schleiermacher G, Michon J, Huon I, et al. Chromosomal CGH identifies patients with a higher risk of relapse in neuroblastoma without MYCN amplification. Br J Cancer 2007;97:238-246.

39 Ambros I, Ambros P. Schwann cells in neuroblastoma. Eur J Cancer 1995;31A:429-434.

40 Ambros I, Zellner A, Roald B, et al. Role of ploidy, chromosome 1p, and Schwann cells in the maturation of neuroblastoma. N Eng J Med 1996;334:1505-1511.

41 Liu S, Tian Y, Chlenski A, et al. Cross-talk between Schwann cells and neuroblasts influences the biology of neuroblastoma xenografts. Am J Pathol 2005;166: 891-900.

42 Coco S, Defferrari R, Scaruffi P, et al. Genome analysis and gene expression profiling of neuroblastoma and ganglioneuroblastoma reveal differences between neuroblastic and Schwannian stromal cells. J Pathol 2005;207:346-357.

43 Mora J, Akram M, Cheung NK, et al. Laser-capture microdissected schwannian and neuroblastic cells in stage 4 neuroblastomas have the same genetic alterations. Med Pediatr Oncol 2000;35:534-537.

44 Mora J, Cheung N, Juan G, et al. Neuroblastic and Schwannian stromal cells of neuroblastoma are derived from a tumoral progenitor cell. Cancer Res 2001;61:6892-6898. 